Table 1.

\begin{tabular}{lcc}
\hline Parameters & $\mathrm{N}$ & $\%$ \\
\hline Oral dryness & 78 & 83,0 \\
$\quad$ Enlargement of parotid salivary glands & 32 & 34,0 \\
Recurrent parotitis & 19 & 20,2 \\
Retention pain & 31 & 33,0 \\
RF positive >2UNL (>30 IU/ml) & 51 & 54,2 \\
anti-SSA (anti-Ro) positive $(>25 \mathrm{IU} / \mathrm{ml})$ & 81 & 86,1 \\
anti-SSB (anti-La) positive $(>25 \mathrm{IU} / \mathrm{ml})$ & 48 & 51,0 \\
ANA $\geq 1: 320$ & 94 & 100,0 \\
SGUS 0 & 8 & 8,5 \\
SGUS 1 & 1 & 1,0 \\
SGUS 2 & 20 & 21,2 \\
SGUS 3 & 65 & 69,1 \\
Stimulated SFT <2,5ml/5 min & 59 & 62,8 \\
Sialectasia on parotid sialography & 90 & 94,7 \\
Punctate sialectasia & 12 & 12,6 \\
Globular sialectasia & 63 & 66,3 \\
Cavitary sialectasia & 10 & 10,5 \\
Distructive sialectasia & 6 & 6,3 \\
FS $\geq 1$ foci/4 mm2 & 83 & 88,2 \\
\end{tabular}

Correlation was calculated for each SGUS (0-3) with clinical and classical methods of examination of the salivary glands. Pronounced changes in SGUS 3 correlated with recurrent parotitis $r=0.22(p=0.03)$, enlargement of parotid salivary glands $r=0.23(p=0.02)$ and cavitary sialectasia $r=0.23(p=0.02)$. Histological changes FS $\geq 1$ correlated with all grades on ultrasound (SGUS $0 r=-0.24$ $(p=0.01)$, SGUS $1 r=0.41(p=0.000027)$, SGUS $2 r=-0.21(p=0.03)$, SGUS $3 r=0.40(p=0.00006))$. The punctate sialectasia correlated with SGUS 1 $r=-0.28(p=0.005)$ and SGUS $0 r=0.45(p=0.000004)$. Stimulated SFT didn't correlate with ultrasound changes.

Conclusion: : Probably, in everyday clinical practice, SGUS can be used as an additional method for salivary gland assessment, given the availability and safety of the method. The most convenient system for assessing the salivary glands, at present, is the OMERACT SS severity scoring system, however, now we don't have a score for the complete differentiation in pSS and lymphoproliferative complications.

References: :

[1] Jousse-Joulin, Sandrine et al. "Video clipassessment of a salivary gland ultrasound scoring system in Sjögren's syndrome using consensual definitions: an OMERACT ultrasound working group reliability exercise." Annals of therheumatic diseases vol. 78,7 (2019): 967-973. doi:10.1136/ annrheumdis-2019-215024

Disclosure of Interests: None declared

DOI: 10.1136/annrheumdis-2021-eular.2455

\begin{tabular}{|l|l|}
\hline AB0322 & ASSOCIATION OF DEPRESSIVE SYMPTOMS \\
ASSESSED BY BDI II WITH PHYSICAL ACTIVITY IN \\
PATIENTS WITH PRIMARY SJÖGREN'S SYNDROME
\end{tabular}

S. Beider ${ }^{1}$, T. Witte ${ }^{1}$, D. Ernst ${ }^{1} .{ }^{1}$ Medical School Hanover, Department of Rheumatology and Immunology, Hannover, Germany

Background: Patients with primary Sjögren's syndrome (pSS) suffer from pain, oral or ocular dryness and fatigue. Such symptoms can have a detrimental impact on health-related quality of life. The prevalence of mental health disorders in patients with pSS is considerably higher than in the general population [1]. Regular physical activity, such as nordic walking, improves aerobic capacity and may reduce reported fatigue [2]. According to current European League Against Rheumatism (EULAR) recommendations, management of pSS concomitant diseases should be evaluated in patients presenting with fatigue and pain, and severity scored using specific tools [3]

Objectives: This study analyses the frequency and severity of depressive symptoms in patients with pSS and their relationship to fatigue and physical activity. Methods: In this monocentric, cross-sectional study, patients with pSS attending our Rheumatology clinic between January 2019 and March 2020 completed standardized questionnaires: Beck's depression inventory second edition (BDI II) and the international physical activity questionnaire short form (IPAQ-SF). Data were analyzed using SPSS 26 (IBM, Armonk, NY, USA). The tests include bivariate and partial correlations and nonparametric Kruskal-Wallis-Test.

Results: In total, 134 patients were included. The majority were female (117/134, $87.3 \%$ ), and the patients median age was 57 [21 - 85] years. Median duration of disease-related symptoms at inclusion was 56 months (range 0-388 months). Physical activity was low in $44.8 \%(n=60)$, moderate in $32.1 \%(n=43)$, and high in $23.1 \%(n=31)$ of patients. Depressive symptoms of varying severity were identified in $76 / 134(56.7 \%)$ of patients. Severe depression occurred in $10 / 76(13.2 \%)$ and moderate depression in $14 / 76(18.4 \%)$ patients. The remaining $52 / 76(68.4 \%)$ patients had minimal or mild depression. Over two-thirds $(67.7 \%)$ of patients reporting high physical activity showed no depression and no one with a high level of physical activity had severe depression. There was a clear association between the BDI II depression score and the level of physical activity $(p=0.003)$ regardless of age and illness duration. However, not all the symptoms assessed by BDI II had the same impact. From 21 items of the BDI II Inventory, symptoms such as loss of energy and fatigue were profoundly relevant, being reported by over $75 \%$ of patients. The main depressive symptoms, which had a strong negative correlation with the level of physical activity were loss of energy $(p<0.001)$, sadness $(p=0.018)$, inability to make decisions $(p=0.007)$ and loss of pleasure $(p=0.004)$. Somatic symptoms of decreased activation such as fatigue $(p=0.026)$ and concentration difficulty $(p=0.013)$ were also significant. The cognitive, self-negative symptoms were not associated with the level of physical activity. Conclusion: A negative correlation of physical activity with fatigue and depression in patients with pSS has been demonstrated. The somatic symptoms of decreased activation played a profound role in this association, while the cog nitive self-negotiation symptoms were not influenced by physical activity in our patients. Such symptoms as fatigue and loss of energy could potentially be reduced by exercises and functional training and consequently decrease the level of depression. An intervention study in this regard would be recommended. REFERENCES:

[1] Cui, Y., et al., Anxiety and depression in primary Sjögren's syndrome: a cross-sectional study. BMC psychiatry, 2018. 18(1): p. 1-8.

[2] Strömbeck, B., E. Theander, and L. Jacobsson, Effects of exercise on aerobic capacity and fatigue in women with primary Sjögren's syndrome. Rheumatology, 2007. 46(5): p. 868-871.

[3] Ramos-Casals, M., et al., EULAR recommendations for the management of Sjögren's syndrome with topical and systemic therapies. Annals of the rheumatic diseases, 2020. 79(1): p. 3-18.

Disclosure of Interests: None declared

DOI: 10.1136/annrheumdis-2021-eular.2647

\section{AB0323 \\ INTIMA-MEDIA THICKNESS IS INDEPENDENTLY ASSOCIATED WITH STROKE IN PRIMARY ANTIPHOSPHOLIPID SYNDROME}

L. D. Azoulay ${ }^{1}$, A. Mathian ${ }^{1}$, F. Cohen ${ }^{1}$, J. Haroche ${ }^{1}$, M. Pha ${ }^{1}$, P. Cherin ${ }^{1}$ P. Cluzel ${ }^{2}$, Z. Amoura'. ${ }^{1}$ Pitié-Salpetrière, Internal Medicine 2, Paris, France; ${ }^{2}$ Pitié-Salpêtrière, Cardiovascular Imaging and Interventionnal Radiology, Paris, France

Background: Compared to secondary antiphospholipid syndrome (APS), little is known on intima-media thickness (IMT) in primary APS. Previous reports suggest that intima-media thickness may be associated with anti-cardiolipin antibodies, anti- $\beta 2$-glycoprotein-1 antibodies, and with stroke in APS ${ }^{1}$.

Objectives: To assess clinical significance of intima-media thickness in primary APS.

Methods: We have included 29 patients with primary APS (defined according to international consensus criteria) seen, at least once, in our tertiary center for a cardiovascular risk assessment from April 2014 to November 2018.

Results: 29 patients were included (females $83 \%$ ). Mean (SD) age at evaluation was 48 years (16.1). Mean APS duration before assessment was 9.1 years (6.2). 27 patients had thrombotic APS (93\%), among which $13(45 \%)$ had a history of arterial thrombosis (12 strokes and 1 myocardial infarction) and $16(55 \%)$ of venous thrombosis. Obstetrical APS was present in 7 patients (24\%). Positive APL antibodies were: anti-cardiolipin antibodies $(\mathrm{aCL}) \mathrm{n}=15(54 \%)$, anti- $\beta 2$-gly-

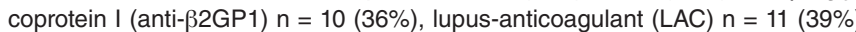
and anti-phospholipid antibodies (aPL) $n=19(68 \%)$. Mean IgG levels (GPL) were 52.4 (80.3) UGPL, 52.2 (124) UI, 39.2 (40.5) UGPL for aCL anti-ßG2P1, and $\mathrm{aPL}$, respectively. 6 patients $(21 \%)$ were simple positive, $3(11 \%)$ were double positive, and $10(36 \%)$ were triple positive.

Cardiovascular risk factors were: smoking $n=6$ patients $(21 \%)$, overweight $n=$ $11(39 \%)$, hypercholesterolemia $n=12(43 \%)$, arterial hypertension $n=11(38 \%)$, chronic kidney disease (defined by estimated glomerular filtration rate $<60 \mathrm{~mL}$ / $\mathrm{mn}) \mathrm{n}=5(17 \%)$. No patient had diabetes mellitus. Median risk SCORE was $0.65(0-2.4)$. Male sex, obesity, chronic kidney disease and risk SCORE were significantly more frequent in patients with arterial APS compared to patients with venous and/or obstetrical APS ( $38 \%$ vs $0 \%, P=0.01 ; 33 \%$ vs $0 \%, P=0.02 ; 38 \%$ vs $0 \%, P=0.01 ; 2.3$ vs $0.7, P=0.03$ ).

22 patients $(76 \%)$ underwent an IMT measurement. Mean IMT was $0.57(0.14)$ millimeters $(\mathrm{mm})$. Median IMT was significantly higher in APS patient with stroke 